\title{
Universally stable adjustment processes - a unifying approach
}

Citation for published version (APA):

Herings, P. J. J. (2000). Universally stable adjustment processes - a unifying approach. METEOR, Maastricht University School of Business and Economics. METEOR Research Memorandum No. 003 https://doi.org/10.26481/umamet.2000003

Document status and date:

Published: 01/01/2000

DOI:

10.26481/umamet.2000003

Document Version:

Publisher's PDF, also known as Version of record

\section{Please check the document version of this publication:}

- A submitted manuscript is the version of the article upon submission and before peer-review. There can be important differences between the submitted version and the official published version of record.

People interested in the research are advised to contact the author for the final version of the publication, or visit the DOI to the publisher's website.

- The final author version and the galley proof are versions of the publication after peer review.

- The final published version features the final layout of the paper including the volume, issue and page numbers.

Link to publication

\footnotetext{
General rights rights.

- You may freely distribute the URL identifying the publication in the public portal. please follow below link for the End User Agreement:

www.umlib.nl/taverne-license

Take down policy

If you believe that this document breaches copyright please contact us at:

repository@maastrichtuniversity.nl

providing details and we will investigate your claim.
}

Copyright and moral rights for the publications made accessible in the public portal are retained by the authors and/or other copyright owners and it is a condition of accessing publications that users recognise and abide by the legal requirements associated with these

- Users may download and print one copy of any publication from the public portal for the purpose of private study or research.

- You may not further distribute the material or use it for any profit-making activity or commercial gain

If the publication is distributed under the terms of Article $25 \mathrm{fa}$ of the Dutch Copyright Act, indicated by the "Taverne" license above, 


\title{
Universally Stable Adjustment Processes - A Unifying Approach -*
}

\author{
P. Jean-Jacques Herings ${ }^{\dagger}$ \\ Department of Economics \\ University of Maastricht
}

February 14, 2000

\begin{abstract}
Both in game theory and in general equilibrium theory there exists a number of universally stable adjustment processes. In game theory these processes typically serve the role of selecting a Nash equilibrium. Examples are the tracing procedure of Harsanyi and Selten or the equilibrium selection procedure proposed by McKelvey and Palfrey. In general equilibrium the processes are adjustment rules by which an auctioneer can clear all markets. Examples are the processes studied by Smale, Kamiya, van der Laan and Talman, and Herings. The underlying reasons for convergence have remained rather mysterious in the literature, and convergence of different processes has seemed unrelated. This paper shows that convergence of all these processes relies on Browder's fixed point theorem.
\end{abstract}

KEYWORDS: Adjustment processes, game theory, general equilibrium, universal convergence.

JEL CODES: C62, C63, C68, C72.

${ }^{*}$ The research of Jean-Jacques Herings has been made possible by a fellowship of the Royal Netherlands Academy of Arts and Sciences.

${ }^{\dagger}$ P.J.J. Herings, Department of Economics, University of Maastricht, P.O. Box 616, 6200 MD Maastricht, The Netherlands.

E-mail: P.Herings@algec.unimaas.nl

Homepage: http://www2.unimaas.nl/ fdewbae/herings.htm 


\section{Introduction}

Both in game theory and in general equilibrium theory there exists a number of adjustment processes that are universally convergent. A universally convergent adjustment process in game theory is an adjustment process that converges to a Nash equilibrium for almost all games. A universally convergent adjustment process in general equilibrium theory is an adjustment process that converges to a Walrasian equilibrium for almost all economies. In game theory these processes typically serve the role of selecting a Nash equilibrium. Examples are the tracing procedure of Harsanyi and Selten (1988) or the equilibrium selection procedure proposed by McKelvey and Palfrey (1995). In general equilibrium the processes are adjustment rules by which an auctioneer can clear all markets. Examples are the processes studied by Smale (1976), van der Laan (1987) and Talman, Kamiya (1990) and Herings (1997a).

There are several reasons to be interested in universally convergent adjustment processes. They give players in a game the opportunity to coordinate on a uniquely determined Nash equilibrium and an auctioneer in an economy to determine a competitive equilibrium price system. In a more decentralized setting, they give rational agents in an economy the possibility to coordinate on current and future prices. Such processes can be used as a tool to compute equilibria, which is also helpful for comparative statics exercises or policy recommendations, see Judd (1997) and Eaves and Schmedders (1999).

In game theory multiplicity of Nash equilibria seems to be the rule rather than the exception. This poses serious problems for Nash equilibrium to be used as a solution concept for games. One way out is to develop a theory that selects a unique equilibrium for any game form, and to suppose that all players adopt that theory. An attempt to make such a theory can be found in Harsanyi and Selten (1988). This theory relies heavily on the tracing procedure as introduced in Harsanyi (1975). The tracing procedure is a strategy adjustment procedure by which players can adopt initial beliefs about the play of their opponents and turn them into uniquely determined beliefs consistent with Nash equilibrium. The surprising aspect of the tracing procedure is that convergence to a Nash equilibrium takes place for almost any game for almost any initial beliefs, so the tracing procedure is universally convergent.

Quantal response equilibria as introduced in McKelvey and Palfrey (1995), are statistical versions of Nash equilibria, where each player's payoff is subject to random error. The concept of equilibrium is consistent in the sense that all player's maximize their utility given the choices made by the others, and the utility maximizing behavior of a player,

together with the error structure, leads to the mixed strategy against which the others optimize. Quantal response equilibria are quite successful in describing the behavior of participants in experiments. McKelvey and Palfrey (1995) also consider a procedure simi- 
lar to the tracing procedure to select a Nash equilibrium. Start with the quantal response equilibrium where choices are completely determined by the error terms, and follow the path of quantal response equilibria that results when the error terms vanish. McKelvey and Palfrey show that for almost all games, a unique Nash equilibrium is selected in this way. Again, universal convergence of the procedure is obtained.

The simplest price adjustment process studied in general equilibrium theory is the Walrasian tatonnement process. It is well-known that it may not converge to a competitive equilibrium, see Scarf (1960) for some examples. The work of Sonnenschein $(1972,1973)$, Mantel (1974) and Debreu (1974), basically claiming that any function satisfying Walras' law is the excess demand function of an economy, makes clear that it is possible to construct many examples where Walrasian tatonnement does not converge and displays highly irregular dynamic behavior. The work of Saari and Simon (1978) and Saari (1985) implies that simple adaptations of the Walrasian tatonnement process will not have better convergence properties. Still, at least three universally convergent price adjustment processes are known in the literature, Smale's global Newton method introduced in Smale (1976), the process of Kamiya (1990), and the process proposed in van der Laan and Talman (1987) for which universal convergence has been shown in Herings (1997a).

The global Newton method of Smale provides a price adjustment rule that does converge to a competitive equilibrium for almost any economy, so universal convergence is the case. But it does not converge for any initial price system. Only when the initial price system is chosen such that the prices of some commodities are sufficiently close to zero, convergence to a competitive equilibrium can be shown. From the work of Keenan (1981) it follows that there may exist an open set of starting price systems for which Smale's process does not converge to some competitive equilibrium price system.

Another universally convergent price adjustment process has been presented in Kamiya (1990). Under rather weak conditions on the total excess demand function, convergence to a competitive equilibrium price system is guaranteed for almost every starting price system. Although the boundary conditions of Kamiya are weak, they are not derived from assumptions on primitive concepts.

An alternative price adjustment process has been proposed in van der Laan and Talman (1987). For this process universal convergence has been shown in Herings (1997a). Under standard conditions on utility functions, consumption sets and initial endowments, this price adjustment process converges to a Walrasian equilibrium price system for almost all economies and almost all starting price systems.

Apparently, several processes in distinct areas of research have been shown to be universally convergent. The reason for these strong convergence properties has remained mysterious up to now, and the convergence proofs were rather ad hoc as a consequence. 
The aim of the current paper is to point out that convergence of each one of these processes can be understood from fixed-point theory and is not even related to differentiability. This makes our proofs very different from the original convergence proofs. It also increases our understanding as to why these distinct adjustment processes converge. This understanding is useful to develop other universally convergent mechanisms that may incorporate features

that are lacking in current processes. For instance, the incorporation of strategic effects of agents that supply and demand commodities in general equilibrium.

Some alternatives and extensions have already been suggested. The procedure descibed in Yamamoto (1993) may serve as an alternative to the tracing procedure, and Joosten and Talman (1997) describe an alternative price adjustment process. Extensions have been made to economies with linear or constant returns to scale production, see van den Elzen (1993, 1997) and van den Elzen, van der Laan and Talman (1994), and to economies with short-run price rigidities, see Herings (1996), Herings, van der Laan, Talman and Venniker (1997), Herings, van der Laan and Venniker (1998), and Herings, van der Laan and Talman (1999). All these extensions can be understood as well from the unifying treatment that is given in this paper.

\section{A Unifying Approach}

Before turning to the specific adjustment processes, it is helpful to highlight the approach that can be used to give a unifying treatment of convergence. Usually, dynamic processes are defined by a system of first-order differential equations

$$
\frac{d x(t)}{d t}=g(x(t))
$$

where $x(t) \in \mathbb{R}^{m}$ denotes the state vector reached at time $t \in \mathbb{R}_{+}$and $g$ is a function from some subset of the state space $\mathbb{R}^{m}$ into $\mathbb{R}^{m}$. The vector $x$ typically corresponds to a mixed strategy combination in case of a strategy adjustment process, and to a price system for a price adjustment process. The function $g$ specifies the way in which players adjust their strategies, or prices adjust in general equilibrium. The initial state $x(0)$ is assumed to be given.

Conditions for which the system of differential equations has a solution are well-known, see for instance Hirsch and Smale (1974). The orbit $\gamma(x(0))$ is the set of state vectors that is generated by the system of first-order differential equations when the initial state is $x(0)$,

$$
\gamma(x(0))=\left\{x \in \mathbb{R}^{m} \mid \exists t \geq 0, x=x(t)\right\} .
$$

We denote the closure of $\gamma(x(0))$ by $\bar{\gamma}(x(0))$, and call $\bar{\gamma}(x(0))$ an orbit as well.

Although all adjustment processes we consider can be formulated as a system of differential equations, they can alternatively be described by the orbit that they generate. 
In fact, all adjustment processes considered share the property that the easiest way to formulate them is in terms of the orbit that they generate. For each adjustment process, we define a system of equations whose solutions correspond to the orbit of the adjustment process. We study the properties of the set of solutions to the system of equations by means of fixed point theory and not by the theory of dynamic systems.

Our assumptions on primitives are so weak, that orbits are not necessarily nicely behaved sets, that is differentiable paths or loops. It is for instance possible that pitchforks may arise, or even higher dimensional solution sets. The way that is usually dealt with such complications is to show that they generically do not occur. For almost all games and for almost all economies that satisfy suitable differentiability conditions, orbits are differentiable paths or loops. We show that as far as convergence is concerned, neither genericity arguments nor differentiability are the main driving forces. Instead, the key to understand convergence comes from an entirely different direction, fixed point theory. In this paper we argue that the convergence of the tracing procedure of Harsanyi and Selten, the selection of a Nash equilibrium by means of quantal response equilibria as proposed by McKelvey and Palfrey, and the convergence of the price adjustment processes of Smale, Kamiya, and van der Laan and Talman, are best understood from a single fixed point theorem that is introduced in Browder (1960).

ThEOREM 2.1 (BROWDER's FIXED POINT THEOREM): Let $S$ be a non-empty, compact, convex subset of $\mathbb{R}^{m}$ and let $\varphi: S \times[0,1] \rightarrow S$ be a continuous function. Then the set $F_{\varphi}=\{(\lambda, s) \in[0,1] \times S \mid s=\varphi(\lambda, s)\}$ contains a connected set $F_{\varphi}^{\mathrm{c}}$ such that $(\{0\} \times S) \cap F_{\varphi}^{c} \neq \emptyset$ and $(\{1\} \times S) \cap F_{\varphi}^{c} \neq \emptyset$.

Theorem 2.1 implies that for all $\lambda \in[0,1],(\{\lambda\} \times S) \cap F_{\varphi} \neq \emptyset$. That property would also follow from a repeated application of the well-known fixed point theorem of Brouwer (1912). The surprising part of the theorem is that there exists a connected set $F_{\varphi}^{c}$ with those properties. Notice that along the connected set of fixed points, it is not necessarily the case that $\lambda$ increases monotonically from 0 to 1 . The value of $\lambda$ increases initially, may decrease later on, and will eventually increase until it reaches the value 1.

\section{The Tracing Procedure of Harsanyi and Selten}

The tracing procedure is used repeatedly in the equilibrium selection theory of Harsanyi and Selten (1988) to find a unique solution of so-called basic games. It is also used to define risk-dominance relationships between Nash equilibria. It models a process of convergent expectations by which rational players will come to adopt and expect each other to adopt on 
a particular Nash equilibrium as the solution for a given game. Before applying the tracing procedure, players are assumed to have a common probability distribution expressing their expectations about the strategy choices of the other players. This common probability distribution is called a prior. In the linear tracing procedure the information on the best replies to the prior is gradually fed back into the expectations of the players. As the linear tracing procedure proceeds, both the prior and the best responses will gradually change until both converge to some Nash equilibrium of the game.

Consider a non-cooperative $N$-person normal form game $\Gamma=\left(\Phi_{1}, \ldots, \Phi_{N}, R_{1}, \ldots, R_{N}\right)$. Each player $i=1, \ldots, N$, has $M_{i}$ pure strategies. The $k$-th pure strategy of player $i$ is denoted by $(i, k)$. The set of pure strategies of player $i$ is denoted by $\Phi_{i}$. The total number of strategies is given by $M=\sum_{i=1}^{N} M_{i}$. The set of all pure strategy combinations is given by $\Phi=\prod_{i=1}^{N} \Phi_{i}$. The function $R_{i}: \Phi \rightarrow \mathbb{R}$ denotes the payoff function of a player $i$ and it is extended in the standard way to the set of all mixed strategy combinations $S=\prod_{i=1}^{N} S^{M_{i}}$. Here we identify all probability distributions on $\Phi_{i}$ with $S^{M_{i}}=\left\{s_{i} \in \mathbb{R}^{M_{i}} \mid \sum_{j=1}^{M_{i}} s_{i j}=1\right\}$. Given a mixed strategy combination $s \in S$ and a mixed strategy $\bar{s}_{i} \in S_{i}$, we denote by $s \backslash \bar{s}_{i}$ the mixed strategy combination that results from replacing $s_{i}$ by $\bar{s}_{i}$. The set of Nash equilibria of $\Gamma$ is denoted $\mathrm{NE}(\Gamma)$.

A probability distribution $s^{0} \in S$, called the prior, is given for the remainder of this paper. The prior describes the initial beliefs of all players about the strategies played by the other players. The prior is assumed to be the same for all players, and the determination of the prior is part of the equilibrium selection theory of Harsanyi and Selten (1988). For every $\lambda \in[0,1]$, the linear tracing procedure generates a Nash equilibrium of the game $\Gamma^{\lambda}=\left(\Phi_{1}, \ldots, \Phi_{N}, H_{1}^{\lambda}, \ldots, H_{N}^{\lambda}\right)$, where the payoff function $H_{i}^{\lambda}: S \rightarrow \mathbb{R}$ of player $i$ is defined by

$$
H_{i}^{\lambda}(s)=\lambda R_{i}(s)+(1-\lambda) R_{i}\left(s^{0} \backslash s_{i}\right) .
$$

The game $\Gamma^{0}$ corresponds to a trivial game, where all players believe that all their opponents play with probability 1 according to the prior beliefs. The game $\Gamma^{1}$ coincides with the game $\Gamma$. The linear tracing procedure links a Nash equilibrium of the game $\Gamma^{0}$, to a Nash equilibrium of $\Gamma^{1}$. Let $\mathcal{L}$ denote the set of all Nash equilibria related to the games $\Gamma^{\lambda}$, $\lambda \in[0,1]$, so

$$
\mathcal{L}=\left\{(\lambda, s) \in[0,1] \times S \mid s \in \mathrm{NE}\left(\Gamma^{\lambda}\right)\right\} .
$$

The linear tracing procedure is said to feasible if there exists a continuous function $\gamma$ : $[0,1] \rightarrow \mathcal{L}$, i.e. a path, such that $\gamma(0) \in \mathcal{L} \cap(\{0\} \times S)$ and $\gamma(1) \in \mathcal{L} \cap(\{1\} \times S)$. The linear tracing procedure is said to be well-defined if there exists a unique way to connect $\gamma(0)$ to $\gamma(1)$ by a continuous function. We consider feasibility as the more important property. Indeed, it is possible to go from feasibility to well-definedness by invoking certain regularity 
properties of $\mathcal{L}$. These regularity properties follow from the theory of differential topology, as $\mathcal{L}$ is a set defined by $M$ equations in $M+1$ unknowns, leaving one degree of freedom for a typical game, see Herings and Peeters (1999) for a rigorous proof.

Since $\mathcal{L}$ is a set that can be described by a finite number of polynomial inequalities, it is a semi-algebraic set. All the components of $\mathcal{L}$, that is all maximally connected subsets of $\mathcal{L}$, are also path-connected. Therefore, any two points in a component of $\mathcal{L}$ can be joined by a path, see for instance Schanuel, Simon, and Zame (1991) for a nice introduction into the properties of semi-algebraic sets. To show that the linear tracing procedure is feasible, it is sufficient to show that $\mathcal{L}$ has a component that intersects both the sets $\{0\} \times S$ and $\{1\} \times S$.

The proof of feasibility of the linear tracing procedure presented here is not new. It coincides with one of the proofs proposed in Herings (1997b). It is repeated here for illustrational purposes, as the connection between Browder's fixed point theorem and the tracing procedure is the closest of all the adjustment processes that we will consider.

Let the function $\sigma_{i}:[0,1] \times S \rightarrow S^{M_{i}}$ be defined by

$$
\sigma_{i}(\lambda, s)=\arg \max _{\bar{s}_{i} \in S^{M_{i}}} \lambda R_{i}\left(s \backslash \bar{s}_{i}\right)+(1-\lambda) R_{i}\left(s^{0} \backslash \bar{s}_{i}\right)-\left\|\bar{s}_{i}-s_{i}\right\|_{2}^{2} .
$$

The function $\sigma_{i}$ is continuous since the penalty $-\left\|\bar{s}_{i}-s_{i}\right\|_{2}^{2}$ is strictly concave in $\bar{s}_{i}$.

We define the function $f:[0,1] \times S \rightarrow S$ by

$$
f(\lambda, s)=\left(\sigma_{1}(\lambda, s), \ldots, \sigma_{N}(\lambda, s)\right) .
$$

The fixed points of $f$ are closely related to the strategies in the set $\mathcal{L}$.

THEOREM 3.1: For any non-cooperative $N$-person game $\Gamma$, for any prior $s^{0}$, it holds that $(\lambda, s) \in \mathcal{L}$ if and only if there is $\lambda \in[0,1]$ such that $f(\lambda, s)=s$.

ProOf: It is obvious that $(\lambda, s) \in \mathcal{L}$ implies $f(\lambda, s)=s$.

Suppose there is $(\bar{\lambda}, \bar{s}) \in[0,1] \times S$ such that $f(\bar{\lambda}, \bar{s})=\bar{s}$, but $(\bar{\lambda}, \bar{s}) \notin \mathcal{L}$. Then, for some $s_{i} \in S^{M_{i}}, H_{i}^{\bar{\lambda}}\left(\bar{s} \backslash s_{i}\right)-H_{i}^{\bar{\lambda}}(\bar{s})=h>0$. Since $H_{i}^{\bar{\lambda}}\left(\bar{s} \backslash s_{i}\right)=\sum_{(i, k) \in \Phi_{i}} s_{i k} H_{i}^{\bar{\lambda}}(\bar{s} \backslash(i, k))$, it holds that, for $0<\varepsilon<1, H_{i}^{\bar{\lambda}}\left(\bar{s} \backslash \varepsilon s_{i}+(1-\varepsilon) \bar{s}_{i}\right)-H_{i}^{\bar{\lambda}}(\bar{s})=\varepsilon h>0$. Now, $\left\|\left(\varepsilon s_{i}+(1-\varepsilon) \bar{s}_{i}\right)-\bar{s}_{i}\right\|^{2}=$ $\varepsilon^{2}\left\|s_{i}-\bar{s}_{i}\right\|^{2}<\varepsilon h$, for small enough $\varepsilon$, contradicting that $\bar{s}_{i}$ is the argument maximizing the expression in the definition of $\sigma_{i}(\bar{\lambda}, \bar{s})$.

Q.E.D.

The argument given in the proof of Theorem 3.1 is the same as the one used in Geanakoplos (1996), where Brouwer's fixed point theorem, as opposed to Kakutani's fixed point theorem, is used to show the existence of a Nash equilibrium in a finite non-cooperative $N$-person game. 
TheOREM 3.2: For any non-cooperative $N$-person game $\Gamma$, for any prior $s^{0}$, the tracing procedure is feasible.

ProOF: It is immediate that $f$ satisfies the conditions of Browder's fixed point theorem and so there is a component $F^{\mathrm{c}}$ of $F=\{(\lambda, s) \in[0,1] \times S \mid s=f(\lambda, s)\}$ such that $(\{0\} \times S) \cap F^{\mathrm{c}} \neq \emptyset$ and $(\{1\} \times S) \cap F^{\mathrm{c}} \neq \emptyset$. By Theorem 3.1 it follows that $F=\mathcal{L}$, so $F^{c}$ is a subset of $\mathcal{L}$ that connects a best response to the prior $s^{0}$ to a Nash equilibrium $s^{*}$. Q.E.D.

Feasibility of the tracing procedure is a corollary to Browder's fixed point theorem.

\section{The Quantal Response Equilibria of McKelvey and Palfrey}

Quantal response equilibria as introduced in McKelvey and Palfrey (1995), are statistical versions of Nash equilibria, where each player's payoff is subject to random error. One possible interpretation is that players make errors according to some random process when calculating their expected payoffs. An alternative interpretation is that players calculate expected payoffs correctly, but have an additive payoff disturbance associated with each available pure strategy. For a given specification of the error structure, a quantal response equilibrium is a mixed strategy combination that is consistent with optimizing behavior subject to the error structure.

Consider a non-cooperative $N$-person normal form game $\Gamma=\left(\Phi_{1}, \ldots, \Phi_{N}, R_{1}, \ldots, R_{N}\right)$. Player $i$ 's payoff when playing pure strategy $(i, k)$ against a mixed strategy combination $s$ is subject to error and is given by

$$
\widehat{R}_{i}(s \backslash(i, k))=R_{i}(s \backslash(i, k))+\varepsilon_{i k} .
$$

Player $i$ 's error vector $\varepsilon_{i}=\left(\varepsilon_{i 1}, \ldots, \varepsilon_{i M_{i}}\right)$ is distributed according to a joint distribution with density function $\phi_{i}$. Given the vector of payoffs that player $i$ receives when playing his pure strategies and when errors are absent, $\bar{R}_{i}=\left(R_{i}(s \backslash(i, 1)), \ldots, R_{i}\left(s \backslash\left(i, M_{i}\right)\right)\right)$ for some $s \in S$, the $i k$-response set $E_{i k}\left(\bar{R}_{i}\right)$ is defined as the set of error vectors that make pure strategy $(i, k)$ the best response, so

$$
E_{i k}\left(\bar{R}_{i}\right)=\left\{\varepsilon_{i} \in \mathbb{R}^{M_{i}} \mid \bar{R}_{i k}+\varepsilon_{i k} \geq \bar{R}_{i j}+\varepsilon_{i j}, j=1, \ldots, M_{i}\right\} .
$$

The probability of choosing pure strategy $(i, k)$ is then given by

$$
\sigma_{i k}\left(\bar{R}_{i}\right)=\int_{\varepsilon_{i} \in E_{i k}\left(\bar{R}_{i}\right)} \phi_{i}\left(\varepsilon_{i}\right) d \varepsilon_{i}
$$


A quantal response equilibrium is a mixed strategy combination $s^{*} \in S$ that is consistent with the error structure, thus

$$
s_{i k}^{*}=\sigma_{i k}\left(R_{i}\left(s^{*} \backslash(i, 1)\right), \ldots, R_{i}\left(s^{*} \backslash\left(i, M_{i}\right)\right)\right), \quad i=1, \ldots, N, k=1, \ldots, M_{i} .
$$

The following specification of the error structure is quite common in the theory of individual choice behavior, see Luce (1959), and leads to the logistic quantal response equilibria. For any parameter $\theta \geq 0$, the logistic quantal response function is defined by

$$
\sigma_{i k}\left(\bar{R}_{i}\right)=\frac{\exp \left(\theta \bar{R}_{i k}\right)}{\sum_{j=1}^{M_{i}} \exp \left(\theta \bar{R}_{i j}\right)}, \quad \bar{R}_{i} \in \mathbb{R}^{M_{i}},
$$

and is obtained when $\phi_{i}$ corresponds to the extreme value distribution. The parameter $\theta$ is inversely related to the error level. When $\theta=0$, the choice of all players is completely determined by the errors, and corresponds to playing all pure strategies with equal probability. When $\theta$ approaches infinity, the influence of the errors disappears. This suggests a way of selecting Nash equilibria analogously to the tracing procedure. Start from the quantal response equilibrium at $\theta=0$ and let the influence of errors go to zero. McKelvey and Palfrey (1995) show that for generic games, this approach selects a unique Nash equilibrium. We show that for all games the quantal response equilibrium at $\theta=0$ is connected by a set of quantal response equilibria to at least one Nash equilibrium.

Given an error level corresponding to $\theta$, the set of quantal response equilibria of $\Gamma$ is denoted $\mathrm{QRE}^{\theta}(\Gamma)$. Let $\mathcal{Q}$ denote the set of all quantal response equilibria for varying values of $\theta \in \mathbb{R}_{+}$, so

$$
\mathcal{Q}=\left\{(\theta, s) \in \mathbb{R}_{+} \times S \mid s \in \mathrm{QRE}^{\theta}(\Gamma)\right\}
$$

or alternatively

$$
\mathcal{Q}=\left\{(\theta, s) \in \mathbb{R}_{+} \times S \mid s_{i k}=\frac{\exp \left(\theta R_{i}(s \backslash(i, k))\right)}{\sum_{j=1}^{M_{i}} \exp \left(\theta R_{i}(s \backslash(i, j))\right)}, \quad i=1, \ldots, N, k=1, \ldots, M_{i}\right\} .
$$

To investigate whether the quantal response equilibrium at $\theta=0$ is connected to a Nash equilibrium, it is useful to make the transformation $\theta=\lambda /(1-\lambda)$ and to define

$$
\widetilde{\mathcal{Q}}=\left\{(\lambda, s) \in[0,1) \times S \mid s_{i k}=\frac{\exp \left(\frac{\lambda}{1-\lambda} R_{i}(s \backslash(i, k))\right)}{\sum_{j=1}^{M_{i}} \exp \left(\frac{\lambda}{1-\lambda} R_{i}(s \backslash(i, j))\right)}, \quad i=1, \ldots, N, k=1, \ldots, M_{i}\right\} .
$$

We define the function $f:[0,1) \times S \rightarrow S$ by

$$
f_{i k}(\lambda, s)=\frac{\exp \left(\frac{\lambda}{1-\lambda} R_{i}(s \backslash(i, k))\right)}{\sum_{j=1}^{M_{i}} \exp \left(\frac{\lambda}{1-\lambda} R_{i}(s \backslash(i, j))\right)}, \quad i=1, \ldots, N, k=1, \ldots, M_{i} .
$$

The fixed points of $f$ are closely related to the strategies in the set $\widetilde{\mathcal{Q}}$. 
TheOrem 4.1: For any non-cooperative $N$-person game $\Gamma$, it holds that $(\lambda, s) \in \widetilde{\mathcal{Q}}$ if and only if there is $\lambda \in[0,1)$ such that $f(\lambda, s)=s$.

ProOf: Obvious.

Q.E.D.

The following result follows immediately from Browder's fixed point theorem, so a proof is omitted.

THEOREM 4.2: For any non-cooperative $N$-person game $\Gamma$, for any $\bar{\lambda} \in(0,1)$, there is a component $\widetilde{\mathcal{Q}}^{\mathrm{c}}$ of $\widetilde{\mathcal{Q}}$ such that $(\{0\} \times S) \cap \mathcal{Q}^{\mathrm{c}} \neq \emptyset$ and $(\{\bar{\lambda}\} \times S) \cap \mathcal{Q}^{\mathrm{c}} \neq \emptyset$.

The theorem makes clear that the unique quantal response equilibrium at $\theta=0$ is connected by quantal response equilibria to a quantal response equilibrium for an arbitrarily high value of $\theta$.

The next step is to extend Theorem 4.2 and to consider what happens in the limit. In particular, we want to show that the quantal response equilibrium at $\theta=0$ is connected by quantal response equilibria to a Nash equilibrium. To this end, we define

$$
\overline{\mathcal{Q}}=\widetilde{\mathcal{Q}} \cup(\{1\} \times \mathrm{NE}(\Gamma))
$$

and we show the following result.

THEOREM 4.3: For any non-cooperative $N$-person game $\Gamma$, there is a component $\overline{\mathcal{Q}}^{\mathrm{c}}$ of $\overline{\mathcal{Q}}$ such that $(\{0\} \times S) \cap \overline{\mathcal{Q}}^{c} \neq \emptyset$ and $(\{1\} \times S) \cap \overline{\mathcal{Q}}^{c} \neq \emptyset$.

Proof: For $n \in \mathbb{N}$, denote the component $\widetilde{\mathcal{Q}}^{\text {c }}$ of $\widetilde{\mathcal{Q}}$ such that $(\{0\} \times S) \cap \mathcal{Q}^{\mathrm{c}} \neq \emptyset$ and $\left(\left\{1-\frac{1}{n}\right\} \times S\right) \cap \mathcal{Q}^{c} \neq \emptyset$ by $\widetilde{Q}^{n}$. Note that, for $n \in \mathbb{N}, \widetilde{Q}^{n} \subset \widetilde{Q}^{n+1}$. By Mas-Colell (1985), Theorem A.5.1.(ii), page 10, the closed limit of the sequence $\widetilde{Q}^{n}$, denoted $\bar{Q}^{c}$, is connected. We show that $\bar{Q}^{c} \subset \bar{Q}$.

Let $(\bar{\lambda}, \bar{s})$ be an element of $\overline{\mathcal{Q}}^{\mathrm{c}}$. Then there exists a sequence of points $\left\{\left(\lambda^{n}, s^{n}\right)\right\}_{n \in \mathbb{N}}$ such that $\lambda^{n}<1, f\left(\lambda^{n}, s^{n}\right)=s^{n}$, and $\left(\lambda^{n}, s^{n}\right) \rightarrow(\bar{\lambda}, \bar{s})$. If $\bar{\lambda}<1$, then the continuity of $f$ implies $(\bar{\lambda}, \bar{s}) \in \widetilde{\mathcal{Q}} \subset \overline{\mathcal{Q}}$. Suppose $\bar{\lambda}=1$, and suppose $\bar{s}$ is not a Nash equilibrium. Then there is a player $i$, a pair of pure strategies $(i, k)$ and $(i, l)$, and $\varepsilon>0$ such that $\bar{s}_{i k}>0$, but $R_{i}(\bar{s} \backslash(i, k))+\varepsilon<R_{i}(\bar{s} \backslash(i, l))$. Since $s^{n} \rightarrow \bar{s}$, there is $\bar{n}$ such that $R_{i}\left(s^{n} \backslash(i, k)\right)+\varepsilon<$ $R_{i}\left(s^{n} \backslash(i, l)\right)$ for all $n \geq \bar{n}$. However,

$$
\begin{aligned}
\lim _{n \rightarrow \infty} f_{i k}\left(\lambda^{n}, s^{n}\right) & =\lim _{n \rightarrow \infty} \frac{\exp \left(\frac{\lambda^{n}}{1-\lambda^{n}} R_{i}\left(s^{n} \backslash(i, k)\right)\right)}{\sum_{j=1}^{M_{i}} \exp \left(\frac{\lambda^{n}}{1-\lambda^{n}} R_{i}\left(s^{n} \backslash(i, j)\right)\right)} \\
& \leq \lim _{n \rightarrow \infty} \frac{\exp \left(\frac{\lambda^{n}}{1-\lambda^{n}} R_{i}\left(s^{n} \backslash(i, k)\right)\right)}{\exp \left(\frac{\lambda^{n}}{1-\lambda^{n}} R_{i}\left(s^{n} \backslash(i, l)\right)\right)}=0 .
\end{aligned}
$$


Therefore,

$$
0<\bar{s}_{i k}=\lim _{n \rightarrow \infty} s_{i k}^{n}=\lim _{n \rightarrow \infty} f_{i k}\left(\lambda^{n}, s^{n}\right)=0,
$$

a contradiction. We have shown that $\overline{\mathcal{Q}}^{\mathrm{c}} \subset \overline{\mathcal{Q}}$.

The property that $(\{0\} \times S) \cap \overline{\mathcal{Q}}^{\mathrm{c}} \neq \emptyset$ and $(\{1\} \times S) \cap \overline{\mathcal{Q}}^{\mathrm{c}} \neq \emptyset$ is immediate. Q.E.D.

As was the case for the tracing procedure, Browder's fixed point theorem is the basic tool needed to show the connectedness of the quantal response equilibrium at $\theta=0$ to a Nash equilibrium.

\section{The Global Newton Method of Smale}

The correspondence with Browder's fixed point theorem is almost immediate for procedures to select Nash equilibria in games. For price adjustment processes, the relationship is a little harder to detect.

There are several versions of Smale's global Newton method. Here we follow the approach suggested in Smale (1976) on page 117 to apply his method to the function

$$
z(p)-\bar{z}(p) \mathbb{1}, \quad p \in \mathbb{R}_{+}^{L} \backslash\{0\},
$$

where $z: \mathbb{R}_{+}^{L} \backslash\{0\} \rightarrow \mathbb{R}^{L}$ is an excess demand function of an economy, $\bar{z}(p)=\sum_{l=1}^{L} z_{l}(p) / L$ is the mean excess demand at $p$, and $\mathbb{1}$ is a vector of ones of appropriate dimension. The zero points of $z(\cdot)$ coincide with the zero points of $z(\cdot)-\bar{z}(\cdot) \mathbb{1 1}$. The following assumption is made throughout this section.

Assumption 1: The function $z: \mathbb{R}_{+}^{L} \backslash\{0\} \rightarrow \mathbb{R}^{L}$ satisfies:

\section{Continuity.}

2. Homogeneity: for every $p \in \mathbb{R}_{+}^{L} \backslash\{0\}$, for all $\lambda>0, z(\lambda p)=z(p)$.

3. Walras' law: for every $p \in \mathbb{R}_{+}^{L} \backslash\{0\}, p \cdot z(p)=0$.

4. Boundary behavior: for every $p \in \mathbb{R}_{+}^{L} \backslash\left(\mathbb{R}_{++}^{L} \cup\{0\}\right), z(p)-\bar{z}(p) \mathbb{1}$ is not radially outward pointing.

Assumption 1 is weak version of the assumption in Smale (1976), where twice continuous differentiability of $z$ is assumed and a rather complicated boundary condition is stated. We follow here the suggestion of Varian (1977) and weaken the boundary condition of Smale 
(1976) to the requirement that $z(p)-\bar{z}(p) \mathbb{1}$ is not radially outward pointing, i.e. there is no $\mu>0$ such that $z(p)-\bar{z}(p) \mathbb{1}=\mu(p-(1 / L) \mathbb{1})$.

The assumed boundary behavior is weaker than the requirement that $z_{l}(p)>0$ for some $l \in L$ for which $p_{l}=0$, a requirement that is natural for a function defined on $\mathbb{R}_{+}^{L} \backslash\{0\}$. Indeed, if $l$ is such that $p_{l}=0$ and $z_{l}(p)>0$, then Walras' law implies that there is $l^{\prime}$ such that $p_{l^{\prime}}>0$ and $z_{l}(p)>z_{l^{\prime}}(p)$. So, $z_{l}(p)-\bar{z}(p)>z_{l^{\prime}}(p)-\bar{z}(p)$, whereas $-1 / L=p_{l}-1 / L<p_{l^{\prime}}-1 / L$, which implies that $z(p)-\bar{z}(p)$ is not radially outward pointing.

By Walras' law we can normalize prices such as to belong to $S^{L}$. The suggestion of Varian $(1977)^{1}$ is to extend $z(p)-\bar{z}(p) \mathbb{1}$ as follows to a function $\widetilde{z}: B^{L} \cap T^{L} \rightarrow \mathbb{R}^{L}$, where

$$
\begin{aligned}
B^{L} & =\left\{p \in \mathbb{R}^{L} \mid \sum_{l=1}^{L}\left(p_{l}-1 / L\right)^{2} \leq 1\right\}, \\
T^{L} & =\left\{p \in \mathbb{R}^{L} \mid \sum_{l=1}^{L} p_{l}=1\right\} .
\end{aligned}
$$

The radial projection on $S^{L}$ is denoted by $\tilde{\pi}$, so if $p \in T^{L} \backslash S^{L}$, and $l^{\prime} \in \arg \min _{l=1, \ldots, L} p_{l}$, then

$$
\tilde{\pi}(p)=\frac{1 / L}{1 / L-p_{l^{\prime}}} p+\frac{-p_{l^{\prime}}}{1 / L-p_{l^{\prime}}}(1 / L) \mathbb{1} .
$$

If $p \in S^{L}$, then $\tilde{\pi}(p)=p$.

The function $\widetilde{z}: B^{L} \cap T^{L} \rightarrow \mathbb{R}^{L}$ is defined by

$$
\widetilde{z}(p)=\widetilde{\pi}(p)-p+\left(1-\|p-(1 / L) \mathbb{1}\|_{2}\right)(z(\widetilde{\pi}(p))-\bar{z}(\widetilde{\pi}(p)) \mathbb{1}), \quad p \in B^{L} \cap T^{L} .
$$

The function $\widetilde{z}$ is a positive multiple of $z(p)-\bar{z}(p) \mathbb{1}$ on $S^{L}$ and is continuous on $B^{L} \cap T^{L}$. The contribution of the term $z(\tilde{\pi}(p))-\bar{z}(\tilde{\pi}(p)) \mathbb{1}$ vanishes on the relative boundary of $B^{L} \cap T^{L}$, where it holds that $\|p-(1 / L) \mathbb{1}\|_{2}=1$. This makes the function $\widetilde{z}$ radially inward pointing on the relative boundary of $B^{L} \cap T^{L}$. The term $\tilde{\pi}(p)-p$ vanishes for $p \in S^{L}$.

Replacing $z(p)-\bar{z}(p) \mathbb{1}$ by $\widetilde{z}(p)$ does not eliminate any zero points. It also doesn't add new zero points. There are no equilibria of $\tilde{z}$ on the relative boundary of $B^{L} \cap T^{L}$ as the term $1-\|p-(1 / L) \mathbb{1}\|_{2}$ vanishes there and the remaining term is $\widetilde{\pi}(p)-p$. Consider a point $p$ not on the relative boundary of $B^{L} \cap T^{L}$ and outside $S^{L}$. Then $\widetilde{z}(p)=0$ if and only if

$$
\tilde{\pi}(p)-p+\left(1-\|p-(1 / L) \mathbb{1}\|_{2}\right)(z(\tilde{\pi}(p))-\bar{z}(\tilde{\pi}(p)) \mathbb{1})=0 .
$$

Then it holds that

$$
\begin{aligned}
z(\widetilde{\pi}(p))-\bar{z}(\tilde{\pi}(p)) \mathbb{1} & =\frac{1}{1-\|p-(1 / L) \mathbb{1}\|_{2}}(p-\tilde{\pi}(p)) \\
& =\frac{-L p_{l^{\prime}}}{1-\|p-(1 / L) \mathbb{1}\|_{2}}(\widetilde{\pi}(p)-(1 / L) \mathbb{1}),
\end{aligned}
$$

\footnotetext{
${ }^{1}$ The construction of Varian(1977) applies to a more abstract problem on the unit disk, but our variation of his construction to the set $S^{L}$ is rather straightforward.
} 
where $l^{\prime} \in \arg \min _{l=1, \ldots, L} p_{l}$. This implies that $z$ is radially outward pointing at $\tilde{\pi}(p)$, a contradiction to Assumption 1.4.

The differential equation of Smale's global Newton method is given by

$$
\begin{aligned}
\partial \widehat{z}(p) \frac{d p}{d t} & =-\lambda(p) \widehat{z}(p), \\
\mathbb{1}^{\top} \frac{d p}{d t} & =0,
\end{aligned}
$$

where $\lambda$ is an arbitrary scalar function of $p$ such that

$$
\operatorname{sign}(\lambda(p))=\operatorname{sign} \operatorname{det} \partial \widehat{z}(p),
$$

and $\widehat{z}$ is the function $\widetilde{z}$ with the last component omitted. Since the sum of the components of $\widetilde{z}(p)$ equals zero, it holds that $\mathbb{1}^{\top} \partial \widetilde{z}(p)=0$. Then $\partial \widehat{z}(p) \frac{d p}{d t}=-\lambda(p) \widehat{z}(p)$ implies $\partial \widetilde{z}_{L}(p) \frac{d p}{d t}=$ $-\lambda(p) \widetilde{z}_{L}(p)$, so the adjustment of the price of commodity $L$ is similar to the adjustment of the prices of other commodities. Since $\mathbb{1}^{\top} \frac{d p}{d t}=0$, the sum of the prices is kept equal to one.

We choose the starting price system $p^{0}$ in the relative boundary of $B^{L} \cap T^{L}$ to guarantee convergence to a competitive equilibrium price system. In Keenan (1981) it has been shown that Smale's process may not converge for starting price systems in the relative interior of $B^{L} \cap T^{L}$.

As Smale (1976) shows, his process generates price systems in the set

$$
P=\left\{p \in B^{L} \cap T^{L} \mid \exists \theta \geq 0, \widetilde{z}(p)=\theta \widetilde{z}\left(p^{0}\right)\right\} .
$$

It is easily verified, by taking $\theta=1$, that $p^{0} \in P$, and, by taking $\theta=0$, that $p^{*} \in P$ if $p^{*} \in S^{L}$ is an equilibrium price system. By the arguments given before there are no solutions for $\theta=0$ with $p^{*} \in\left(B^{L} \cap T^{L}\right) \backslash S^{L}$. From the definition of the set $P$ it follows that the differential equation adjusts prices in such a way that the excess demand remains proportional to the excess demand at the starting price system.

Under suitable differentiability assumptions, for a generic economy, Smale (1976) shows that the component of $P$ containing $p^{0}$ is a path that connects $p^{0}$ to a Walrasian equilibrium price system. We show that even without such differentiability assumptions, and without restricting attention to generic economies, the component of $P$ containing $p^{0}$ connects $p^{0}$ to a Walrasian equilibrium price system. Smale's global Newton method is said to be convergent if this latter property holds.

For $\lambda \geq 0$, we define the set

$$
T^{L}(\lambda)=\left\{p \in B^{L} \cap T^{L} \mid p \cdot \widetilde{z}\left(p^{0}\right) \leq(1-2 \lambda) p^{0} \cdot \widetilde{z}\left(p^{0}\right)\right\} .
$$

In Figure 1 the set $T^{L}(\lambda)$ is shown for various values of $\lambda$. The set $T^{L}(0)$ contains only the point $p^{0}$. The set $T^{L}(\lambda)$ expands when $\lambda$ increases. The set $T^{L}(1)$ equals $B^{L} \cap T^{L}$. For 


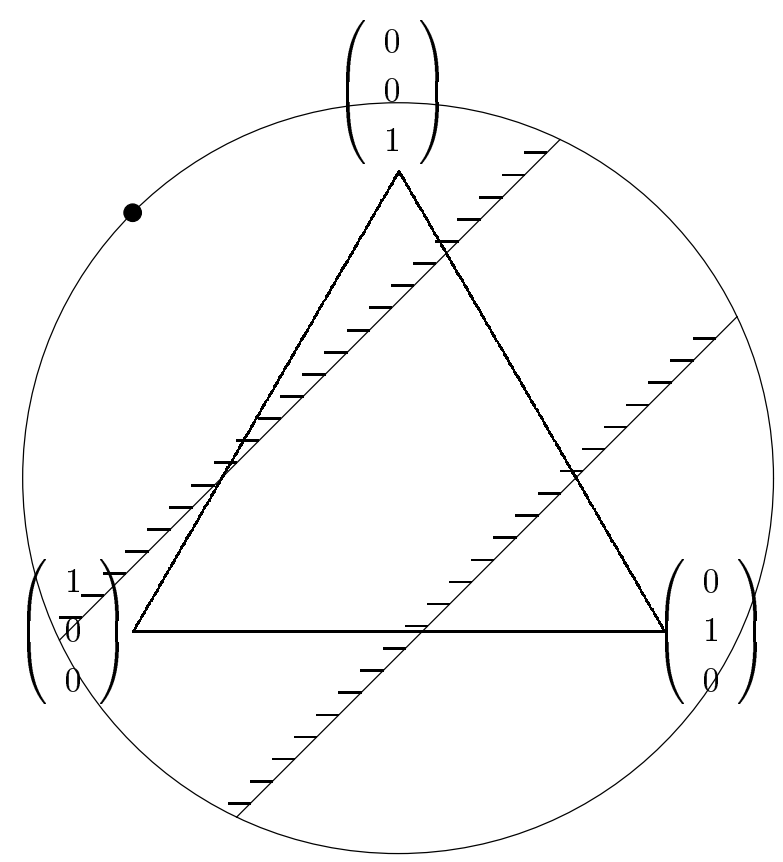

Figure 1. The sets $S^{L}, T^{L}(0), T^{L}(1 / 3), T^{L}(2 / 3)$ and $T^{L}(1)$, for $p^{0}=(0.545,-0.455,0.91)^{\top}$.

$p \in B^{L} \cap T^{L}$, we define $\lambda(p)=\left(p-p^{0}\right) \cdot \widetilde{z}\left(p^{0}\right) /-2 p^{0} \cdot \widetilde{z}\left(p^{0}\right)$. It holds that $p \in T^{L}(\lambda)$ if and only if $\lambda \geq \lambda(p)$.

For any non-empty, closed, convex subset $X$ of $\mathbb{R}^{m}$, the continuous function $\pi_{X}: \mathbb{R}^{m} \rightarrow$ $X$ is the orthogonal projection on $X$, so $\pi_{X}(y)=x$ if $x \in X$ and $\|y-x\|_{2} \leq\|y-\bar{x}\|_{2}$, for all $\bar{x} \in X$. We define the function $f:[0,1] \times T^{L}(1) \rightarrow T^{L}(1)$ by

$$
f(\lambda, p)=\pi_{T^{L}(\lambda)}(p+\widetilde{z}(p)) .
$$

The fixed points of $f$ coincide with the prices in the set $P$.

TheOREm 5.1: For any excess demand function z satisfying Assumption 1, for any $p^{0}$ in the relative boundary of $B^{L} \cap T^{L}$, it holds that $p \in P$ if and only if there is $\lambda \in[0,1]$ such that $f(\lambda, p)=p$. Moreover, either $\widetilde{z}(p) \neq 0$ and $\lambda=\lambda(p)$ or $\widetilde{z}(p)=0$ and $f(\lambda, p)=p$ for all $\lambda \geq \lambda(p)$.

Proof: Consider a fixed point $\bar{p}$ of $f(\bar{\lambda}, \cdot)$, so $\bar{p}=f(\bar{\lambda}, \bar{p})=\pi_{T^{L}(\bar{\lambda})}(\bar{p}+\widetilde{z}(\bar{p}))$. We show that $\bar{p} \in P$.

Since $f(0, \bar{p})=p^{0}$, it is obvious that $f(0, \bar{p})=\bar{p}$ implies $\bar{p}=p^{0}$, so $\bar{p} \in P$. Next consider the case $\bar{\lambda}>0$. For $\bar{p}$ in the relative boundary of $B^{L} \cap T^{L}, \widetilde{z}(\bar{p})$ is radially inward pointing, so obviously $\pi_{T^{L}(\bar{\lambda})}(\bar{p}+\widetilde{z}(\bar{p})) \neq \bar{p}$. Consider $\bar{p}$ in the relative interior of $B^{L} \cap T^{L}$. Then $\bar{p}=\pi_{T^{L}(\bar{\lambda})}(\bar{p}+\widetilde{z}(\bar{p}))$ if and only if the projection of $\bar{p}+\widetilde{z}(\bar{p})$ on $\widetilde{T}^{L}(\lambda)=\left\{p \in T^{L} \mid\right.$ $\left.p \cdot \widetilde{z}\left(p^{0}\right) \leq(1-2 \bar{\lambda}) p^{0} \cdot \widetilde{z}\left(p^{0}\right)\right\}$ equals $\bar{p}$. 
The projection of an arbitrary vector $x$ on the set $\widetilde{T}^{L}(\lambda)$ is determined by the following optimization problem.

$$
\min _{y \in \mathbb{R}^{L}} \sum_{l=1}^{L} 1 / 2\left(y_{l}-x_{l}\right)^{2} \text { s.t. }(1-2 \lambda) p^{0} \cdot \widetilde{z}\left(p^{0}\right)-y \cdot \widetilde{z}\left(p^{0}\right) \geq 0 .
$$

The necessary and sufficient Kuhn-Tucker conditions for an optimum are given by

$$
\begin{aligned}
y-x+\mu \widetilde{z}\left(p^{0}\right) & =0 \\
\mu\left((1-2 \lambda) p^{0} \cdot \widetilde{z}\left(p^{0}\right)-y \cdot \widetilde{z}\left(p^{0}\right)\right) & =0 \\
(1-2 \lambda) p^{0} \cdot \widetilde{z}\left(p^{0}\right)-y \cdot \widetilde{z}\left(p^{0}\right) & \geq 0 \\
\mu & \geq 0
\end{aligned}
$$

where $y$ equals the projection $\pi_{\widetilde{T}^{L}(\lambda)}(x)$ and $\mu$ denotes the shadow price of the constraint $(1-2 \lambda) p^{0} \cdot \widetilde{z}\left(p^{0}\right)-y \cdot \widetilde{z}\left(p^{0}\right) \geq 0$.

Since $\bar{p}=\pi_{T^{L}(\bar{\lambda})}(\bar{p}+\widetilde{z}(\bar{p}))$, it follows that there exists $\mu \geq 0$ such that

$$
\bar{p}-\bar{p}-\widetilde{z}(\bar{p})+\mu \widetilde{z}\left(p^{0}\right)=0,
$$

so $\widetilde{z}(\bar{p})=\mu \widetilde{z}\left(p^{0}\right)$, and $\bar{p} \in P$. This completes the first part of the proof.

Consider some $\bar{p} \in P$. If $\widetilde{z}(\bar{p})=0$, then it is trivially the case that $f(\lambda, \bar{p})=\bar{p}$ whenever $\bar{p} \in T^{L}(\lambda)$, i.e. when $\lambda \geq \lambda(\bar{p})$. Suppose $\widetilde{z}(\bar{p}) \neq 0$. It is obvious that $f(\lambda, \bar{p}) \neq \bar{p}$ when $\lambda \neq \lambda(\bar{p})$. It remains to be shown that $f(\lambda(\bar{p}), \bar{p})=\bar{p}$. If $\lambda(\bar{p})=0$, then $\bar{p}=p^{0}$ and trivially $f\left(0, p^{0}\right)=p^{0}$. Suppose $\widetilde{z}(\bar{p}) \neq 0$ and $\lambda(\bar{p})>0$. There exists $\theta>0$ such that $\tilde{z}(\bar{p})=\theta \widetilde{z}\left(p^{0}\right)$. From the necessary and sufficient Kuhn-Tucker conditions it follows that $\pi_{\widetilde{T}^{L}(\lambda(\bar{p}))}(\bar{p}+\widetilde{z}(\bar{p}))=\bar{p}$. Since $\bar{p} \in T^{L}(\lambda(\bar{p})) \subset \widetilde{T}^{L}(\lambda(\bar{p}))$, it holds as well that $\pi_{T^{L}(\lambda(\bar{p}))}(\bar{p}+\tilde{z}(\bar{p}))=\bar{p}$.

Q.E.D.

When $p^{*}$ is a competitive equilibrium price system, then $p^{*}$ is a fixed point of $f$ for any value of $\lambda$ exceeding $\lambda\left(p^{*}\right)$. When $p$ is a price system generated by the adjustment process, but does not correspond to a competitive equilibrium, then $p$ is a fixed point of $f(\lambda(p), \cdot)$, where $\lambda(p)<1$.

At $p^{0}$ the value of $\lambda(\cdot)$ is zero. Along the path of the adjustment process, the value of $\lambda(\cdot)$ increases initially, but it may decrease later on. Eventually, it will increase until it reaches the value 1 , and a competitive equilibrium has been found.

THEOREM 5.2: The price adjustment process converges for any excess demand function satisfying Assumption 1, for any $p^{0}$ in the relative boundary of $B^{L} \cap T^{L}$.

PROOF: It is immediate that $f$ satisfies the conditions of Browder's fixed point theorem and so there is a component $F^{c}$ of $F=\left\{(\lambda, p) \in[0,1] \times T^{L}(1) \mid p=f(\lambda, p)\right\}$ such that 
$\left(\{0\} \times T^{L}(1)\right) \cap F^{\mathrm{c}} \neq \emptyset$ and $\left(\{1\} \times T^{L}(1)\right) \cap F^{\mathrm{c}} \neq \emptyset$. By Theorem 5.1 it follows that $F=P$, so $F^{\mathrm{c}}$ is a subset of $P$ that connects the starting price system $p^{0}$ to some competitive equilibrium price system $p^{*}$.

Q.E.D.

The proof of Theorem 5.2 show that convergence of the price adjustment process is a corollary to Browder's fixed point theorem.

\section{The Price Adjustment Process of Kamiya}

In Kamiya (1990) the prices of commodities are normalized by assuming that $\sum_{l=1}^{L}\left(p_{l}\right)^{2}=1$. An adjustment process is defined for a total excess demand function $z: \mathbb{R}_{+}^{L} \backslash\{0\} \rightarrow \mathbb{R}^{L}$ and a starting price system $p^{0} \in \mathbb{R}_{++}^{L}$ with $\sum_{l=1}^{L}\left(p_{l}^{0}\right)^{2}=1$. The following assumption is made throughout this section.

Assumption 2: The function $z: \mathbb{R}_{+}^{L} \backslash\{0\} \rightarrow \mathbb{R}^{L}$ satisfies:

1. Continuity.

2. Homogeneity: for every $p \in \mathbb{R}_{+}^{L} \backslash\{0\}$, for all $\lambda>0, z(\lambda p)=z(p)$.

3. Walras' law: for every $p \in \mathbb{R}_{+}^{L} \backslash\{0\}, p \cdot z(p)=0$.

4. Boundary behavior: for $p \in \mathbb{R}_{+}^{L} \backslash\{0\}$, for $l=1, \ldots, L, p_{l}=0$ implies $z_{l}(p)>0$.

Assumption 2 is a weak version of the assumptions in Kamiya (1990), where also twice continuous differentiability of $z$ on $\mathbb{R}_{++}^{L}$ is assumed. Using the normalization discussed above and Walras' law, we can replace the excess demand function $z$ by the excess demand function $\widehat{z}: \dot{B}_{+}^{L-1} \rightarrow \mathbb{R}^{L-1}$, where

$$
\dot{B}_{+}^{L-1}=\left\{\widehat{p} \in \mathbb{R}_{+}^{L-1} \mid \sum_{l=1}^{L-1}\left(\widehat{p}_{l}\right)^{2}<1\right\}
$$

and $\widehat{z}_{l}(\widehat{p})=z_{l}\left(\widehat{p}_{1}, \ldots, \widehat{p}_{L-1}, \sqrt{1-\sum_{l=1}^{L-1}\left(\widehat{p}_{l}\right)^{2}}\right), l=1, \ldots, L-1$. The function $\widehat{z}$ is obtained from omitting the last component of $z$ and making use of the price normalization.

Kamiya's process is a weighted average of Smale's global Newton method, $\partial \widehat{z}(\widehat{p}) \frac{d \widehat{p}}{d t}=$ $-\lambda(\widehat{p}) \widehat{z}(\widehat{p})$, and Walrasian tatonnement, $\frac{d \widehat{p}}{d t}=\widehat{z}(\widehat{p})$. The weights depend on the norm of the excess demand and the distance between $\widehat{p}$ and the initial price system $\widehat{p}^{0}$, where $\widehat{p}^{0}$ denotes the initial price system with component $L$ left out. When formulated as a differential equation, Kamiya's process is given by

$$
\left(\frac{\partial \widehat{z}(\widehat{p})}{\|\widehat{z}(\widehat{p})\|_{2}}-\frac{I}{\left\|\widehat{p}-\widehat{p}^{0}\right\|_{2}}\right) \frac{d \widehat{p}}{d t}=-\lambda(\widehat{p}) \widehat{z}(\widehat{p}),
$$


where $I$ is the $(L-1) \times(L-1)$ identity matrix and $\lambda$ is an arbitrary scalar function of $\hat{p}$ such that

$$
\operatorname{sign}(\lambda(\widehat{p}))=\operatorname{sign} \operatorname{det}\left(\frac{I}{\left\|\widehat{p}-\widehat{p}^{0}\right\|_{2}}-\frac{\partial \widehat{z}(\widehat{p})}{\|\widehat{z}(\widehat{p})\|_{2}}\right) .
$$

Although $d \widehat{p} / d t$ is not directly defined at $\widehat{p}=\widehat{p}^{0}$ or for a competitive equilibrium price system $\hat{p}$, it can be appropriately defined by taking a limit. The process corresponds to Walrasian tatonnement at $\widehat{p}^{0}$, and it becomes Smale's global Newton method as it approaches an equilibrium.

As Kamiya (1990) shows, prices generated by the differential equation belong to the set

$$
\begin{aligned}
P=\left\{\widehat{p} \in \mathbb{R}_{+}^{L-1} \mid\right. & \sum_{l=1}^{L-1}\left(\widehat{p}_{l}\right)^{2}<1 \\
& \left.\exists \theta \in[0,1], \text { for } l=1, \ldots, L-1, \theta \widehat{z}_{l}(\widehat{p})=(1-\theta)\left(\widehat{p}_{l}-p_{l}^{0}\right)\right\} .
\end{aligned}
$$

It is easily verified that $\theta=0$ yields $p=p^{0}$ as the unique solution, so $p^{0} \in P$. By considering $\theta=1$ it follows that if $p^{*}$ is a Walrasian equilibrium price system with $\sum_{l=1}^{L}\left(p_{l}^{*}\right)^{2}=1$, then $\left(p_{1}^{*}, \ldots, p_{L-1}^{*}\right) \in P$. From the definition of the set $P$ it follows that the differential equation adjusts prices in such a way that the excess demand at a price system is proportional to the difference between this price system and the initial price system.

Kamiya (1990) shows that under suitable differentiability assumptions, for a generic economy, the component of $P$ containing $p^{0}$ is a path that connects $p^{0}$ to a Walrasian equilibrium price system. We show that even without differentiability assumptions, and without restricting attention to generic economies, the component of $P$ containing $p^{0}$ connects $p^{0}$ to a Walrasian equilibrium price system. Kamiya's adjustment process is said to be convergent if this latter property holds.

It follows from the boundary behavior and the continuity of $z$ that there exists $\varepsilon>0$, $\varepsilon \leq p_{L}^{0}$, such that $z_{L}(p)>0$ whenever $p_{L} \leq \varepsilon$ and $\sum_{l=1}^{L}\left(p_{l}\right)^{2}=1$. We introduce the set

$$
B_{+}^{L-1}(\varepsilon)=\left\{\hat{p} \in \mathbb{R}_{+}^{L-1} \mid \sum_{l=1}^{L-1}\left(\widehat{p}_{l}\right)^{2} \leq 1-\varepsilon\right\}
$$

and extend the excess demand function $\widehat{z}$ to a function $\widetilde{z}$ defined on $\mathbb{R}^{L-1}$ by setting

$$
\widetilde{z}(\widehat{p})=\widehat{z}\left(\pi_{B_{+}^{L-1}(\varepsilon)}(\widehat{p})\right), \quad \widehat{p} \in \mathbb{R}^{L-1} .
$$

We define the set $\widetilde{P}$ by omitting the non-negativity constraints on prices in $P$ and replacing $\widehat{z}(\widehat{p})$ by $\widetilde{z}(\widehat{p})$, so

$$
\widetilde{P}=\left\{\widehat{p} \in \mathbb{R}^{L-1} \mid \exists \theta \in[0,1], \text { for } l=1, \ldots, L-1, \theta \widetilde{z}_{l}(\widehat{p})=(1-\theta)\left(\widehat{p}_{l}-p_{l}^{0}\right)\right\} .
$$

Lemma 6.1: For any excess demand function z satisfying Assumption 2, for any $p^{0} \in \mathbb{R}_{++}^{L}$ with $\sum_{l=1}^{L}\left(p_{l}^{0}\right)^{2}=1$, it holds that $P=\widetilde{P}$. 
Proof: Consider some $\hat{p} \in P$. First, it is shown that $\widehat{p} \in B_{+}^{L-1}(\varepsilon)$. Suppose not, then $0<\sqrt{1-\sum_{l=1}^{L-1}\left(\widehat{p}_{l}\right)^{2}}<\varepsilon$, so $z_{L}\left(\widehat{p}_{1}, \ldots, \widehat{p}_{L-1}, \sqrt{1-\sum_{l=1}^{L-1}\left(\widehat{p}_{l}\right)^{2}}\right)>0$. By Walras's law it follows that

$$
0>\sum_{l=1}^{L-1} \widehat{p}_{l} \widehat{z}_{l}(\widehat{p})
$$

Since $\widehat{p} \in P$ there is $\theta \in[0,1]$ such that $\theta \widehat{z}_{l}(\widehat{p})=(1-\theta)\left(\widehat{p}_{l}-p_{l}^{0}\right), l=1, \ldots, L-1$. If $\theta=0$, then $\widehat{p}=\widehat{p}^{0}$, which implies $\sqrt{1-\sum_{l=1}^{L-1}\left(\widehat{p}_{l}\right)^{2}}=p_{L}^{0} \geq \varepsilon$, contradicting our supposition. If $\theta=1$, then $\widehat{z}(\widehat{p})=0$, which contradicts $0>\sum_{l=1}^{L-1} \widehat{p}_{l} \widehat{z}_{l}(\widehat{p})$. It follows that $\theta \in(0,1)$. But then

$$
\sum_{l=1}^{L-1} \widehat{p}_{l} \widehat{z}_{l}(\widehat{p})=\frac{1-\theta}{\theta} \sum_{l=1}^{L-1} \widehat{p}_{l}\left(\widehat{p}_{l}-p_{l}^{0}\right)>0,
$$

where the inequality comes from $\widehat{p}^{0} \neq \widehat{p}, \sum_{l=1}^{L-1} p_{l}^{0} \leq \sum_{l=1}^{L-1} \widehat{p}_{l}$, and $\theta<1$. This contradicts $0>\sum_{l=1}^{L-1} \widehat{p}_{l} \widehat{z}_{l}(\widehat{p})$. Consequently, $\hat{p} \in B_{+}^{L-1}(\varepsilon)$, from which it is obtained that $\widetilde{z}(\widehat{p})=\widehat{z}(\widehat{p})$ and $\widehat{p} \in \widetilde{P}$.

Now consider some $\widehat{p} \in \widetilde{P}$. Suppose $\widehat{p} \notin B_{+}^{L-1}(\varepsilon)$. Denote the projection $\pi_{B_{+}^{L-1}(\varepsilon)}(\widehat{p})$ by $\widehat{\pi}$. Then there is $l^{\prime}$ such that $\widehat{\pi}_{l^{\prime}}=0$ or $\sum_{l=1}^{L-1} \widehat{\pi}_{l}=1-\varepsilon$. In the first case it holds that $\widehat{p}_{l^{\prime}} \leq 0$ and $\widetilde{z}_{l^{\prime}}(\widehat{p})>0$ and, for some $\theta \in(0,1){ }^{2}$

$$
0<\theta \widetilde{z}_{l^{\prime}}(\widehat{p})=(1-\theta)\left(\widehat{p}_{l^{\prime}}-p_{l^{\prime}}^{0}\right)<0
$$

a contradiction. In the latter case it holds that $0>\sum_{l=1}^{L-1} \widehat{\pi}_{l} \widetilde{z}_{l}(\widehat{p})$ and, for some $\theta \in(0,1)$, $\theta \widetilde{z}_{l}(\widehat{p})=(1-\theta)\left(\widehat{p}_{l}-p_{l}^{0}\right), l=1, \ldots, L-1$. Because of the first case, we may assume without loss of generality that $\hat{\pi} \gg 0$. Then it follows from the properties of the projection that $\widehat{p}=\lambda \widehat{\pi}$ for some $\lambda>1$. Therefore,

$$
0>\sum_{l=1}^{L-1} \widehat{\pi}_{l} \widetilde{z}_{l}(\widehat{p})=\lambda \sum_{l=1}^{L-1} \widehat{p}_{l} \widetilde{z}_{l}(\widehat{p})=\lambda \frac{1-\theta}{\theta} \sum_{l=1}^{L-1} \widehat{p}_{l}\left(\widehat{p}_{l}-p_{l}^{0}\right)>0,
$$

a contradiction. Consequently, $\widehat{p} \in B_{+}^{L-1}(\varepsilon)$, from which it is obtained that $\widetilde{z}(\widehat{p})=\widehat{z}(\widehat{p})$ and $\widehat{p} \in P$.

Q.E.D.

The theorem makes clear that we may either study the set $P$ or the set $\widetilde{P}$ in order to study the adjustment process.

For $\lambda \geq 0$, we define the set

$$
T^{L-1}(\lambda)=\left\{\widehat{p} \in \mathbb{R}^{L-1} \mid\left\|\widehat{p}-\widehat{p}^{0}\right\|_{2} \leq \lambda\right\} .
$$

In Figure 2 the set $T^{L-1}(\lambda)$ is shown for various values of $\lambda$. The set $T^{L-1}(0)$ contains only the point $p^{0}$. The set $T^{L-1}(\lambda)$ expands when $\lambda$ increases, and $T^{L-1}(1)$ contains the set 


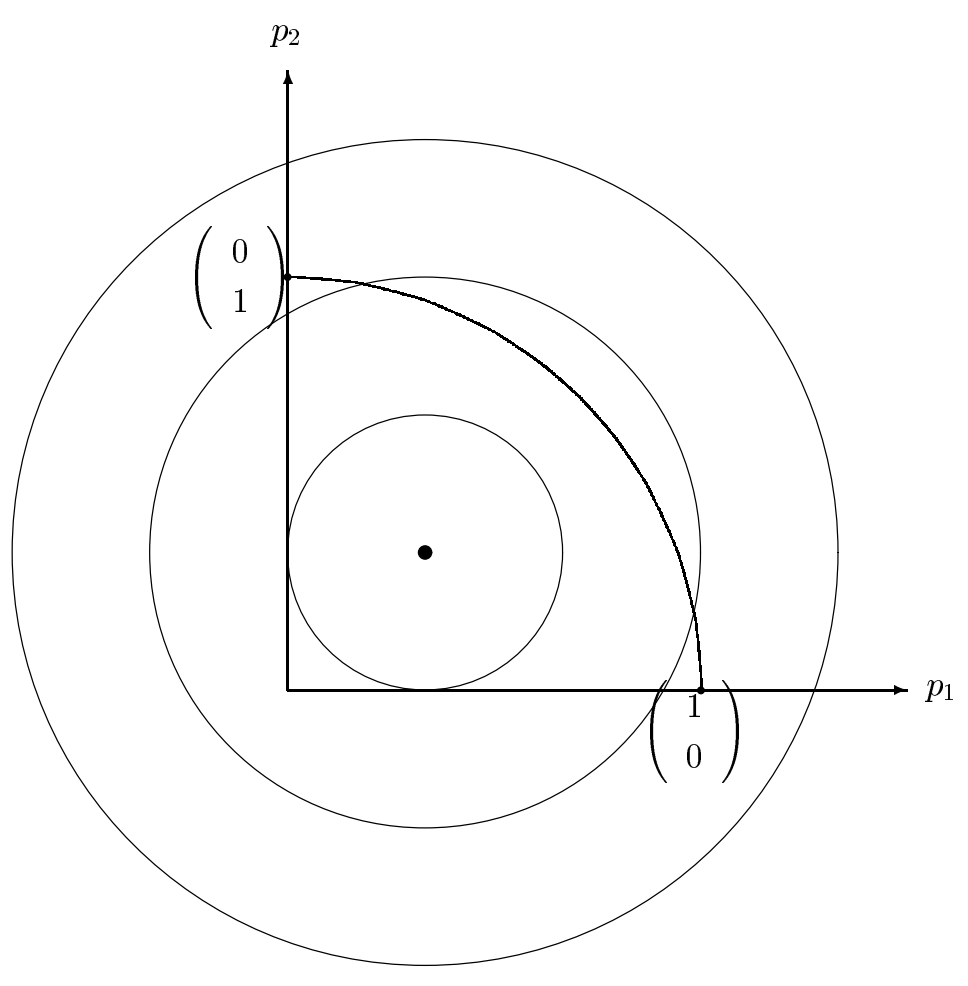

Figure 2 . The sets $\dot{B}_{+}^{L-1}, T^{L-1}(0), T^{L-1}(1 / 3), T^{L-1}(2 / 3)$ and $T^{L-1}(1)$, for $p^{0}=(1 / 3,1 / 3,1 / 3)^{\top}$.

$\dot{B}_{+}^{L-1}$. For $\widehat{p} \in \mathbb{R}^{L-1}$, we define $\lambda(\widehat{p})$ as the distance to $\widehat{p}^{0}, \lambda(\widehat{p})=\left\|\widehat{p}-\widehat{p}^{0}\right\|_{2}$. It is immediate that $\widehat{p} \in T^{L-1}(\lambda)$ for all $\lambda \geq \lambda(\widehat{p})$.

We define the function $f:[0,1] \times T^{L-1}(1) \rightarrow T^{L-1}(1)$ by

$$
f(\lambda, p)=\pi_{T^{L-1}(\lambda)}(p+\widetilde{z}(p)) .
$$

The fixed points of $f$ coincide with the prices in the set $P$.

THEOREM 6.2: For any excess demand function z satisfying Assumption 2, for any $p^{0}$ with $\sum_{l=1}^{L}\left(p_{l}^{0}\right)^{2}=1$, it holds that $\hat{p} \in P$ if and only if there is $\lambda \in[0,1]$ such that $f(\lambda, \hat{p})=\hat{p}$. Moreover, either $\widehat{z}(\widehat{p}) \neq 0$ and $\lambda=\lambda(\widehat{p})$ or $z(\widehat{p})=0$ and $f(\lambda, \hat{p})=\hat{p}$ for all $\lambda \geq \lambda(\widehat{p})$.

Proof: Consider a fixed point $\widehat{p}$ of $f(\lambda, \cdot)$, so $\widehat{p}=f(\lambda, \widehat{p})=\pi_{T^{L-1}(\lambda)}(\widehat{p}+\widetilde{z}(\widehat{p}))$. We show that $\widehat{p} \in \widetilde{P}$, from which it follows that $\widehat{p} \in P$ by Lemma 6.1.

Since $f(0, \widehat{p})=\widehat{p}^{0}$, it is obvious that $f(0, \widehat{p})=\widehat{p}$ implies $\widehat{p}=\widehat{p}^{0}$, so $\widehat{p} \in P$.

Consider the case $\lambda>0$. The projection of an arbitrary vector $x$ on the set $T^{L-1}(\lambda)$ is

\footnotetext{
${ }^{2}$ The argument that $\theta \in(0,1)$ is similar to the one in the first part of this proof.
} 
determined by the following optimization problem.

$$
\min _{y \in \mathbb{R}^{L-1}} \sum_{l=1}^{L-1} 1 / 2\left(y_{l}-x_{l}\right)^{2} \text { s.t. } \lambda^{2}-\sum_{l=1}^{L-1}\left(y_{l}-p_{l}^{0}\right)^{2} \geq 0 .
$$

The necessary and sufficient Kuhn-Tucker conditions for an optimum are given by

$$
\begin{aligned}
y_{l}-x_{l}+2 \mu\left(y_{l}-p_{l}^{0}\right) & =0, \quad l=1, \ldots, L-1, \\
\mu\left(\lambda^{2}-\sum_{l=1}^{L-1}\left(y_{l}-p_{l}^{0}\right)^{2}\right) & =0, \\
\lambda^{2}-\sum_{l=1}^{L-1}\left(y_{l}-p_{l}^{0}\right)^{2} & \geq 0, \\
\mu & \geq 0,
\end{aligned}
$$

where $y$ equals the projection $\pi_{T^{L-1}(\lambda)}(x)$ and $\mu$ denotes the shadow price of the constraint $\lambda^{2}-\sum_{l=1}^{L-1}\left(y_{l}-p_{l}^{0}\right)^{2} \geq 0$.

It follows that there exists $\mu \geq 0$ such that

$$
\widetilde{z}_{l}(\widehat{p})=2 \mu\left(\widehat{p}_{l}-p_{l}^{0}\right), \quad l=1, \ldots, L-1
$$

Since $\mu \geq 0$, it follows that $\widehat{p} \in P$.

Consider some $\widehat{p} \in P$, so $\widehat{p} \in \widetilde{P}$ by Lemma 6.1 . If $\widehat{z}(\widehat{p})=0$, then it is trivially the case that $f(\lambda, \widehat{p})=\widehat{p}$ for all $\lambda \geq \lambda(\widehat{p})$. Suppose $\widehat{z}(\widehat{p}) \neq 0$. If $\lambda(\widehat{p})=0$, then $\widehat{p}=\widehat{p}^{0}$ and trivially $f\left(0, \widehat{p}^{0}\right)=\widehat{p}^{0}$. Suppose $\widehat{z}(\widehat{p}) \neq 0$ and $\lambda(\widehat{p})>0$. We need to show that $f(\lambda(\widehat{p}), \widehat{p})=\widehat{p}$, which is equivalent to the statement that the projection of $\widehat{p}+\widetilde{z}(\widehat{p})$ on $T^{L-1}(\lambda(\widehat{p}))$ equals $\widehat{p}$.

Since $\lambda(\widehat{p})>0$ there exists $\theta \in(0,1)$ such that $\theta \widetilde{z}_{l}(p)=(1-\theta)\left(p_{l}-p_{l}^{0}\right), l=1, \ldots, L-1$. Substitute in the Kuhn-Tucker conditions, $y_{l}=\widehat{p}_{l}, x_{l}=\widehat{p}_{l}+\widetilde{z}_{l}(\widehat{p}), \mu=\frac{\theta}{2(1-\theta)}$ and observe that all equalities and inequalities in the Kuhn-Tucker conditions are satisfied. Q.E.D.

When $p^{*}$ is a competitive equilibrium, then $\left(p_{1}^{*}, \ldots, p_{L-1}^{*}\right)$ is a fixed point of $f$ for any value of $\lambda$ exceeding $\lambda\left(p_{1}^{*}, \ldots, p_{L-1}^{*}\right)$. When $\widehat{p}$ is a price system generated by the adjustment process, but does not correspond to a competitive equilibrium, then $\hat{p}$ is a fixed point of $f(\lambda(\widehat{p}), \cdot)$.

At $\hat{p}^{0}$ the value of $\lambda(\cdot)$ is zero. Along the path of the adjustment process, the value of $\lambda(\cdot)$ increases initially, but it may decrease later on. Eventually, it will increase until it reaches the value 1 , and a competitive equilibrium has been found.

THEOREM 6.3: The price adjustment process converges for any excess demand function satisfying Assumption 2, for any $p^{0} \in \mathbb{R}_{++}^{L}$ with $\sum_{l=1}^{L}\left(p_{l}^{0}\right)^{2}=1$. 
Proof: It is immediate that $f$ satisfies the conditions of Browder's fixed point theorem and so there is a component $F^{c}$ of $F=\left\{(\lambda, \widehat{p}) \in[0,1] \times T^{L-1}(1) \mid \widehat{p}=f(\lambda, \widehat{p})\right\}$ such that $\left(\{0\} \times T^{L-1}(1)\right) \cap F^{c} \neq \emptyset$ and $\left(\{1\} \times T^{L-1}(1)\right) \cap F^{c} \neq \emptyset$. By Theorem 6.2 it follows that $F=P$, so $F^{\mathrm{c}}$ is a subset of $P$ that connects the starting price system $\hat{p}^{0}$ to some competitive equilibrium price system $\widehat{p}^{*}$.

Q.E.D.

Convergence of the price adjustment process is a corollary to Browder's fixed point theorem.

\section{The Price Adjustment Process of van der Laan and Talman}

Van der Laan and Talman (1987) introduce a price adjustment process for an exchange economy. The prices of the commodities are normalized by $\sum_{l=1}^{L} p_{l}=1$. Given a total excess demand function $z: \mathbb{R}_{++}^{L} \rightarrow \mathbb{R}^{L}$ and a starting price system $p^{0} \in \mathbb{R}_{++}^{L}$ with $\sum_{l=1}^{L} p_{l}^{0}=1$, the adjustment process generates price systems in the set

$$
\begin{aligned}
P=\left\{p \in \mathbb{R}_{++}^{L} \mid\right. & \sum_{l=1}^{L} p_{l}=1, \\
& \text { for } l^{\prime}=1, \ldots, L, \quad z_{l^{\prime}}(p)<0 \Rightarrow \frac{p_{l^{\prime}}}{p_{l^{\prime}}^{0}}=\min _{l=1, \ldots, L} \frac{p_{l}}{p_{l}^{0}} \\
& \text { for } \left.l^{\prime}=1, \ldots, L, \quad z_{l^{\prime}}(p)>0 \Rightarrow \frac{p_{l^{\prime}}}{p_{l^{\prime}}^{0}}=\max _{l=1, \ldots, L} \frac{p_{l}}{p_{l}^{0}}\right\} .
\end{aligned}
$$

Two types of restrictions are made on prices in the set $P$. The first is an innocuous price normalization, $\sum_{l=1}^{L} p_{l}=1$. The second concerns the requirement that the relative price of a commodity, i.e. the ratio of the price of a commodity and its initial price, be minimal when the commodity is in positive excess supply, and maximal when the commodity is in positive excess demand. This is closely related to the ideas behind Walrasian tatonnement, where prices of commodities in positive excess supply are decreased and those of commodities in positive excess demand are increased. It is obvious that the starting price system $p^{0}$ belongs to $P$. It can also be verified that whenever $p^{*}$ is a Walrasian equilibrium price system with $\sum_{l=1}^{L} p_{l}^{*}=1$, then $p^{*} \in P$.

In Herings (1997) it is shown that, under suitable differentiability assumptions, for a generic economy, the component of $P$ containing $p^{0}$ is a path that connects $p^{0}$ to a Walrasian equilibrium price system. We show that even without such differentiability assumptions, and without restricting attention to generic economies, the component of $P$ containing $p^{0}$ connects $p^{0}$ to a Walrasian equilibrium price system. The adjustment process is said to be convergent if this latter property holds.

To simplify the exposition, we renormalize the units of measurement of quantities of 
commodities to make sure that $p^{0}=(1 / 3,1 / 3,1 / 3)$. It follows that the set $P$ is given by

$$
\begin{aligned}
P=\left\{p \in \mathbb{R}_{++}^{L} \mid\right. & \sum_{l=1}^{L} p_{l}=1, \\
& \text { for } l^{\prime}=1, \ldots, L, \quad z_{l^{\prime}}(p)<0 \Rightarrow p_{l^{\prime}}=\min _{l=1, \ldots, L} p_{l}, \\
& \text { for } \left.l^{\prime}=1, \ldots, L, \quad z_{l^{\prime}}(p)>0 \Rightarrow p_{l^{\prime}}=\max _{l=1, \ldots, L} p_{l}\right\} .
\end{aligned}
$$

We may also take the value of excess demand $v(p)$, defined by a function $v: \mathbb{R}_{++}^{L} \rightarrow \mathbb{R}^{L}$, where

$$
v_{l}(p)=p_{l} z_{l}(p), \quad l=1, \ldots, L,
$$

instead of the excess demand $z(p)$, to define the set $P$. Since $v(p)$ is positive (negative) if and only if $z(p)$ is positive (negative), it follows that replacing $z(p)$ by $v(p)$ leaves the set $P$ unchanged.

We assume that $z: \mathbb{R}_{++}^{L} \rightarrow \mathbb{R}^{L}$ is an excess demand function, so it satisfies Assumption 3 .

Assumption 3: The function $z: \mathbb{R}_{++}^{L} \rightarrow \mathbb{R}^{L}$ satisfies:

\section{Continuity.}

2. Homogeneity: for every $p \in \mathbb{R}_{++}^{L}$, for all $\lambda>0, z(\lambda p)=z(p)$.

3. Walras' law: for every $p \in \mathbb{R}_{++}^{L}, p \cdot z(p)=0$.

4. Boundary behavior: when $\left(p^{n}\right)_{n \in \mathbb{N}}$ is a sequence converging to $\bar{p} \in \mathbb{R}_{+}^{L} \backslash\{0\}$, then $\lim _{n \rightarrow \infty}\left\|z\left(p^{n}\right)\right\|_{\infty}=+\infty$.

Contrary to Assumptions 1 and 2, Assumption 3 follows from standard assumptions on primitives, that is from standard assumptions on consumption sets, utility functions, and initial endowments.

The continuity and the boundary behavior of $z$ imply that we can choose $\varepsilon>0$ such that for any $p \in S^{L}(\varepsilon)=\left\{p \in S^{L} \mid p_{l} \geq \varepsilon, \quad l=1, \ldots, L\right\}$, it holds that $z_{l}(p)>0$ for some $l$ with $0<p_{l} \leq \varepsilon$.

We modify the value function $v$ near the boundary of $S^{L}$ and extend it to a function $\widetilde{v}$ defined on $T^{L}=\left\{p \in \mathbb{R}^{L} \mid \sum_{l=1}^{L} p_{l}=1\right\}$ by setting

$$
\widetilde{v}(p)=v\left(\pi_{S^{L}(\varepsilon)}(p)\right), \quad p \in T^{L} .
$$

We define the set $\widetilde{P}$ by omitting non-negativity constraints and replacing $z(p)$ by $\widetilde{v}(p)$, so

$$
\begin{aligned}
\widetilde{P}=\left\{p \in T^{L} \mid\right. & \text { for } l^{\prime}=1, \ldots, L, \quad \widetilde{v}_{l^{\prime}}(p)<0 \Rightarrow p_{l^{\prime}}=\min _{l=1, \ldots, L} p_{l}, \\
& \text { for } \left.l^{\prime}=1, \ldots, L, \quad \widetilde{v}_{l^{\prime}}(p)>0 \Rightarrow p_{l^{\prime}}=\max _{l=1, \ldots, L} p_{l}\right\} .
\end{aligned}
$$


Lemma 7.1: For any excess demand function z satisfying Assumption 3, it holds that $P=\widetilde{P}$.

Proof: Consider some $\bar{p} \in P$. It is immediate that $\bar{p} \in S^{L}(\varepsilon)$ and $\widetilde{v}(\bar{p})=v(\bar{p})$. Therefore, $\widetilde{v}_{l}(p)>0$ if and only if $z_{l}(p)>0$ and $\widetilde{v}_{l}(p)<0$ if and only if $z_{l}(p)<0$. So, $\bar{p} \in \widetilde{P}$.

Consider some $\bar{p} \in \widetilde{P}$. Suppose $\bar{p} \notin S^{L}(\varepsilon)$. There is $l^{\prime}$ such that $\pi_{S^{L}(\varepsilon)_{l^{\prime}}}(\bar{p})=\varepsilon$ and $z_{l^{\prime}}\left(\pi_{S^{L}(\varepsilon)}(\bar{p})\right)>0$. But then $\widetilde{v}_{l^{\prime}}(\bar{p})>0$ and $\bar{p}_{l^{\prime}}<\max _{l=1, \ldots, L} \bar{p}_{l}$, a contradiction to $\bar{p} \in \widetilde{P}$. Consequently, $\bar{p} \in S^{L}(\varepsilon)$. Therefore, $\widetilde{v}_{l}(p)>0$ if and only if $z_{l}(p)>0$ and $\widetilde{v}_{l}(p)<0$ if and only if $z_{l}(p)<0$. So, $\bar{p} \in P$.

Q.E.D.

For $\lambda \geq 0$, we define the set

$$
T^{L}(\lambda)=\left\{p \in T^{L} \mid p_{k}-p_{l} \leq \lambda, \quad k, l=1, \ldots, L, k \neq l\right\} .
$$

In Figure 3 the set $T^{L}(\lambda)$ is shown for various values of $\lambda$. The set $T^{L}(0)$ contains only the point $(1 / 3,1 / 3,1 / 3)$. The set $T^{L}(\lambda)$ expands when $\lambda$ increases. The set $T^{L}(1)$ contains the set $S^{L}$.

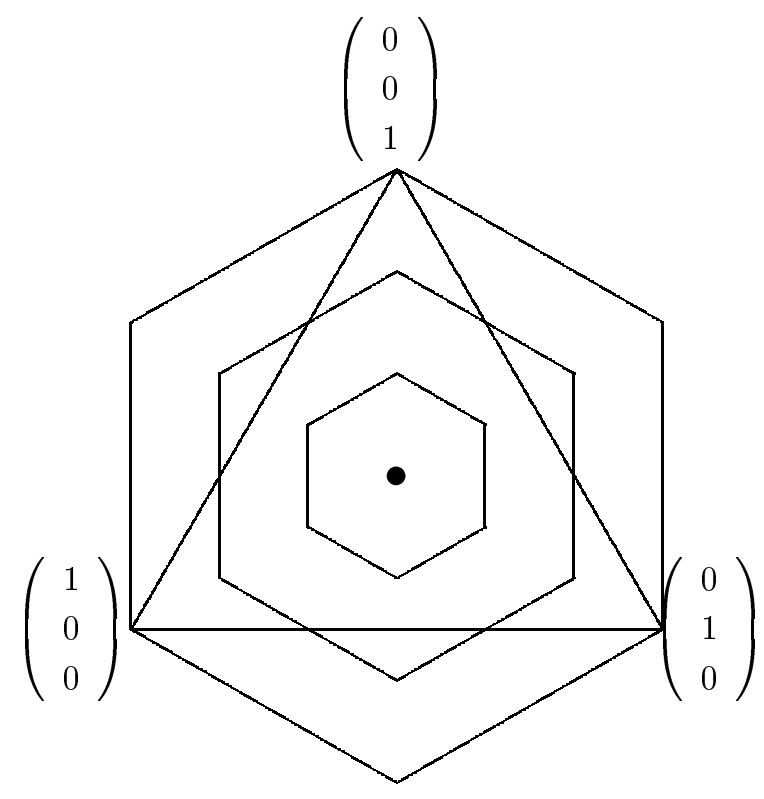

Figure 3 . The sets $S^{L}, T^{L}(0), T^{L}(1 / 3), T^{L}(2 / 3)$ and $T^{L}(1)$.

For $p \in \mathbb{R}^{L}$, we define $\lambda(p)=\max _{k \neq l} p_{k}-p_{l}$. It is immediate that $p \in T^{L}(\lambda)$ for all $\lambda \geq \lambda(p)$. We define the function $f:[0,1] \times T^{L}(1) \rightarrow T^{L}(1)$ by

$$
f(\lambda, p)=\pi_{T^{L}(\lambda)}(p+\widetilde{v}(p)) .
$$


The fixed points of $f$ coincide with the prices in the set $P$.

THEOREM 7.2: For any excess demand function $z$ satisfying Assumption 3, it holds that $p \in P$ if and only if there is $\lambda \in[0,1]$ such that $f(\lambda, p)=p$. Moreover, either $z(p) \neq 0$ and $\lambda=\lambda(p)$, or $z(p)=0$ and $f(\lambda, p)=p$ for all $\lambda \geq \lambda(p)$.

Proof: Consider a fixed point $\bar{p}$ of $f(\bar{\lambda}, \cdot)$, so $\bar{p}=f(\bar{\lambda}, \bar{p})=\pi_{T^{L}(\bar{\lambda})}(\bar{p}+\widetilde{v}(\bar{p}))$. We show that $\bar{p} \in \widetilde{P}$, from which it follows that $\bar{p} \in P$ by Lemma 7.1.

The projection of an arbitrary vector $x$ on the set $T^{L}(\lambda)$ is determined by the following optimization problem.

$$
\begin{aligned}
\min _{y \in \mathbb{R}^{L}} \sum_{l=1}^{L} 1 / 2\left(y_{l}-x_{l}\right)^{2} \text { s.t. } & \sum_{l=1}^{L} y_{l}-1=0, \\
& y_{k}-y_{l}-\lambda \geq 0, \quad k \neq l .
\end{aligned}
$$

The necessary and sufficient Kuhn-Tucker conditions for an optimum are given by

$$
\begin{aligned}
y_{l} & =x_{l}+\mu-\sum_{k \neq l} \mu_{l, k}+\sum_{k \neq l} \mu_{k, l}, \quad l=1, \ldots, L, \\
\sum_{l=1}^{L} y_{l}-1 & =0, \\
\mu_{k, l}\left(y_{k}-y_{l}-\lambda\right) & =0, \quad k \neq l, \\
y_{k}-y_{l}-\lambda & \geq 0, \quad k \neq l, \\
\mu_{k, l} & \geq 0, \quad k \neq l,
\end{aligned}
$$

where $y$ equals the projection $\pi_{T^{L}(\lambda)}(x), \mu$ denotes the shadow price of the constraint $\sum_{l=1}^{L} y_{l}-1=0$, and $\mu_{k, l}, k \neq l$, denotes the shadow price of the constraint $y_{k}-y_{l}-\lambda \geq 0$.

Since $\bar{p}=\pi_{T^{L}(\bar{\lambda})}(\bar{p}+\widetilde{v}(\bar{p}))$, it follows that there exists $\mu \in \mathbb{R}$ and $\mu_{k, l} \geq 0, k \neq l$, such that

$$
\widetilde{v}_{l}(\bar{p})=-\mu+\sum_{k \neq l} \mu_{l, k}-\sum_{k \neq l} \mu_{k, l}, \quad l=1, \ldots, L .
$$

Moreover,

$$
\begin{aligned}
1 & =\sum_{l=1}^{L} \bar{p}_{l}=\sum_{l=1}^{L}\left(\bar{p}_{l}+\widetilde{v}_{l}(\bar{p})+\mu-\sum_{k \neq l} \mu_{l, k}+\sum_{k \neq l} \mu_{k, l}\right) \\
& =1+\sum_{l=1}^{L} \widetilde{v}_{l}(\bar{p})+L \mu+\sum_{l=1}^{L}\left(-\sum_{k \neq l} \mu_{l, k}+\sum_{k \neq l} \mu_{k, l}\right) \\
& =1+L \mu,
\end{aligned}
$$


so $\mu=0$ and

$$
\widetilde{v}_{l}(\bar{p})=\sum_{k \neq l} \mu_{l, k}-\sum_{k \neq l} \mu_{k, l}, \quad l=1, \ldots, L .
$$

It also holds that

$$
\mu_{k, l}\left(\bar{p}_{k}-\bar{p}_{l}-\bar{\lambda}\right)=0, \quad k \neq l .
$$

Suppose $\widetilde{v}_{l^{\prime}}(\bar{p})<0$ for some $l^{\prime}$. Then $\mu_{k, l^{\prime}}>0$ for some $k$, so $\bar{p}_{l^{\prime}}=\bar{p}_{k}-\bar{\lambda}$. Since for all $l, \bar{p}_{l} \geq \bar{p}_{k}-\bar{\lambda}$, it holds that $\bar{p}_{l^{\prime}}=\min _{l=1, \ldots, L} \bar{p}_{l}$. Similarly it can be shown that $\widetilde{v}_{l^{\prime}}(\bar{p})>0$ implies $\bar{p}_{l^{\prime}}=\max _{l=1, \ldots, L} \bar{p}_{l}$. Consequently, it holds that $\bar{p} \in \widetilde{P}$.

Consider some $\bar{p} \in P$, so $\bar{p} \in \widetilde{P}$ by Lemma 7.1. If $z(\bar{p})=0$, then it is trivially the case that $f(\lambda, \bar{p})=\bar{p}$ for all $\lambda \geq \lambda(\bar{p})$. Suppose $z(\bar{p}) \neq 0$. We need to show that $f(\lambda(\bar{p}), \bar{p})=\bar{p}$, which implies that the projection of $\bar{p}+\widetilde{v}(\bar{p})$ on $T^{L}(\bar{\lambda})$ equals $\bar{p}$. This is achieved by substituting in the Kuhn-Tucker conditions $y_{l}=\bar{p}_{l}, x_{l}=\bar{p}_{l}+\widetilde{v}_{l}(\bar{p}), \mu=0$, $\lambda=\lambda(\bar{p}), \mu_{k, l}=\widetilde{v}_{k}(\bar{p}) \widetilde{v}_{l}(\bar{p}) / v$ if $\widetilde{v}_{k}(\bar{p})>0$ and $\widetilde{v}_{l}(\bar{p})<0$, and $\mu_{k, l}=0$, otherwise, where $v=\sum_{\left\{l \mid \widetilde{v}_{l}(\bar{p})<0\right\}} \widetilde{v}_{l}(\bar{p})$. Observe that all equalities and inequalities in the Kuhn-Tucker conditions are satisfied. Q.E.D.

When $p^{*}$ is a competitive equilibrium, then $p^{*}$ is a fixed point of $f$ for any value of $\lambda$ exceeding $\lambda\left(p^{*}\right)$. When $p$ is a price system generated by the adjustment process, but not a competitive equilibrium, then $p$ is a fixed point of $f(\lambda(p), \cdot)$.

At $p^{0}$ the value of $\lambda(\cdot)$ is zero. Along the path of the adjustment process, the value of $\lambda(\cdot)$ increases initially, but it may decrease later on. Eventually, it will increase until it reaches the value 1 , and a competitive equilibrium has been found.

THEOREM 7.3: The price adjustment process converges for any excess demand function satisfying Assumption 3.

Proof: It is immediate that $f$ satisfies the conditions of Browder's fixed point theorem and so there is a component $F^{c}$ of $F=\left\{(\lambda, p) \in[0,1] \times T^{L}(1) \mid p=f(\lambda, p)\right\}$ such that $\left(\{0\} \times T^{L}(1)\right) \cap F^{c} \neq \emptyset$ and $\left(\{1\} \times T^{L}(1)\right) \cap F^{c} \neq \emptyset$. By Theorem 7.2 it follows that $F=P$, so $F^{\mathrm{c}}$ is a subset of $P$ that connects the starting price system $p^{0}$ to some competitive equilibrium price system $p^{*}$.

Q.E.D.

Once again, the convergence of a price adjustment process is intimately connected to Browder's fixed point theorem. 


\section{Conclusion}

We have studied the convergence of a number of distinct adjustment processes in game theory and in general equilibrium theory. Convergence of the processes has been shown before in the literature by rather ad hoc arguments, and only for generic games and generic economies, under suitable differentiability assumptions. We have argued that the driving force behind convergence is to be found in Browder's fixed point theorem, which applies under very general conditions and does not involve any assumptions on differentiability. It is remarkable that not only existence of equilibrium, but also universal stability, is fundamentally based on fixed point theory. The use of Browder's result provides a uniform and simple way to show convergence of all the adjustment processes considered. It also enables us to design a sheer unlimited number of new adjustment processes, that are universally convergent.

\section{References}

Blume, L.E., and W.R. Zame (1992), "The Algebraic Geometry of Competitive Equilibrium," in W. Neuefeind and R.G. Riezman (eds.), Economic Theory and International Trade, Essays in Memoriam J. Trout Rader, Springer-Verlag, Berlin, 53-66.

Brouwer, L.E.J. (1912), “Über Abbildung von Mannigfaltigkeiten,” Mathematische Annalen, 71, 97-115.

Browder, F.E. (1960), "On Continuity of Fixed Points under Deformations of Continuous Mappings," Summa Brasiliensis Mathematicae, 4, 183-191.

Debreu, G. (1974), "Excess Demand Functions," Journal of Mathematical Economics, 1, 15-21.

Eaves, B.C., and K. Schmedders (1999), "General Equilibrium Models and Homotopy Methods," Journal of Economic Dynamics and Control, 23, 1249-1279.

Elzen, A.H. van den (1993), Adjustment Processes for Exchange Economies and Noncooperative Games, Lecture Notes in Economics and Mathematical Systems, 402, Springer-Verlag, Berlin.

Elzen, A.H. van den (1997), "An Adjustment Process for the Standard Arrow/Debreu Model with Production," Journal of Mathematical Economics, 27, 315-324.

Elzen, A.H. van den, G. van der Laan, and A.J.J. Talman (1994), "An Adjustment Process for an Economy with Linear Production Technologies," Mathematics of Operations Research, 19, 341-351. 
Geanakoplos, J.D. (1996), Nash and Walras Equilibrium Via Brouwer, Cowles Foundation Discussion Paper No. 1131, New Haven, Connecticut, 1-18.

Harsanyi, J.C. (1975), "The Tracing Procedure: A Bayesian Approach to Defining a Solution for $n$-Person Noncooperative Games," International Journal of Game Theory, 4, 61-94.

Harsanyi, J.C., and R. Selten (1988), A General Theory of Equilibrium Selection in Games, MIT Press, Cambridge.

Herings, P.J.J. (1996), Static and Dynamic Aspects of General Disequilibrium Theory, Theory and Decision Library, Series C: Game Theory, Mathematical Programming and Operations Research, Kluwer Academic Publishers, Norwell, Massachusetts.

Herings, P.J.J. (1997a), "A Globally and Universally Stable Price Adjustment Process," Journal of Mathematical Economics, 27, 163-193.

Herings, P.J.J. (1997b), "Two Simple Proofs of the Feasibility of the Linear Tracing Procedure," CentER Discussion Paper 9777, CentER, Tilburg University, Tilburg. Forthcoming in Economic Theory.

Herings, P.J.J., G. van der Laan, and A.J.J. Talman (1999), "Price-Quantity Adjustment in a Keynesian Economy," in P.J.J. Herings, G. van der Laan, and A.J.J. Talman (eds.), The Theory of Markets, NorthHolland, Amsterdam, 27-57.

Herings, P.J.J., G. van der Laan, A.J.J. Talman, and R. Venniker (1997), "Equilibrium Adjustment of Disequilibrium Prices," Journal of Mathematical Economics, 27, 53-77.

Herings, P.J.J., G. van der Laan, and R.J.G. Venniker (1998), "The Transition from a Drèze Equilibrium to a Walrasian Equilibrium," Journal of Mathematical Economics, 29, 303-330.

Herings, P.J.J., and R. Peeters (1999), "A Differentiable Homotopy to Compute Nash Equilibria of $n$ Person Games," METEOR Research Memorandum 99/33, University of Maastricht, pp. 1-29.

Hirsch, M.W., and S. Smale (1974), Differential Equations, Dynamical Systems, and Linear Algebra, Academic Press, New York.

Joosten, R.A.M.G., and A.J.J. Talman (1997), "A Globally Convergent Price Adjustment Process for Exchange Economies," Journal of Mathematical Economics, 29, 15-26.

Judd, K.L. (1997), "Computational Economics and Economic Theory: Subsitutes or Complements ?" 
Journal of Economic Dynamics and Control, 21, 907-942.

Kamiya, K. (1990), “A Globally Stable Price Adjustment Process,” Econometrica, 58, 1481-1485.

Keenan, D.C. (1981), "Further Remarks on the Global Newton Method," Journal of Mathematical Economics, 8, 159-165.

Laan, G. van der, and A.J.J. Talman (1987), "A Convergent Price Adjustment Process," Economics Letters, 23, 119-123.

Luce, R.D. (1959), Individual Choice Behaviour: a Theoretical Analysis, Wiley, New York.

Mantel, R.R. (1974), "On the Characterization of Aggregate Excess Demand," Journal of Economic Theory, 7, 348-353.

McKelvey, R.D., and T.R. Palfrey (1995), "Quantal Response Equilibria for Normal Form Games," Games and Economic Behavior, 10, 6-38.

Saari, D.G. (1985), "Iterative Price Mechanisms," Econometrica, 53, 1117-1131.

Saari, D.G., and C.P. Simon (1978), "Effective Price Mechanisms," Econometrica, 46, 1097-1125.

Scarf, H. (1960), "Some Examples of Global Instability of the Competitive Equilibrium," International Economic Review, 1, 157-172.

Schanuel, S.H., L.K. Simon, and W.R. Zame (1991), "The Algebraic Geometry of Games and the Tracing Procedure," in R. Selten (ed.), Game Equilibrium Models II: Methods, Morals and Markets, SpringerVerlag, Berlin, 9-43.

Smale, S. (1976), "A Convergent Process of Price Adjustment and Global Newton Methods," Journal of Mathematical Economics, 3, 107-120.

Sonnenschein, H. (1972), "Market Excess Demand Functions," Econometrica, 40, 549-563.

Sonnenschein, H. (1973), "Do Walras' Identity and Continuity Characterize the Class of Community Excess Demand Functions?," Journal of Economic Theory, 6, 345-354.

Yamamoto, Y. (1993), "A Path-Following Procedure to Find a Proper Equilibrium of Finite Games," International Journal of Game Theory, 22, 249-259. 
Varian, H.R. (1977), "A Remark on Boundary Restrictions in the Global Newton Method," Journal of Mathematical Economics, 4, 127-130. 